\title{
AQUISIÇÃO DE TEMPOS DE VIDA DE FOSFORESCÊNCIA COM RESOLUÇÃO DE FASE E SUA APLICAÇÃO AO ESTUDO DE RELAXAÇÕES EM POLÍMEROS
}

\author{
M. Talhavini e T. D. Z. Atvars* \\ Instituto de Química - Unicamp - CP 6154 - 13083-970 - Campinas - SP
}

Recebido em 17/3/97; aceito em 9/8/97

\begin{abstract}
PHOSPHORESCENCE LIFETIME IN POLYMER RELAXATION STUDIES: PHASE RESOLUTION METHOD. We describe in this work a simple experimental set up to perform time dependent luminescence experiments in time scales from mili-seconds to seconds, based on the phase resolution of the emission signal. This system is composed by modulation of a continuous light source with an external chopper controlled by a lock-in amplifier. We exemplified the utility of the system in studies of phosphorecence emission using benzophenone dissolved in polystyrene and since the phosphorescence intensity and lifetime are temperature-dependent processes, we also studied polymer relaxation processes in the temperature range from 20 to $400 \mathrm{~K}$. A software that drives the spectrofluorimeter and controls both the cryosystem and the lock-in amplifier was developed.
\end{abstract}

Keywords: phase resolution lifetime determinations; benzophenone; polymer relaxation processes.

\section{INTRODUÇÃO}

A grande maioria das moléculas existentes encontra-se, à temperatura ambiente ou abaixo desta, em seu estado eletrônico fundamental, devido à ausência de energia térmica suficiente para a sua excitação eletrônica. Assim a maneira mais usual de se obter moléculas em estados eletrônicos excitados é a utilização de radiação eletromagnética na região do UV/Visível ou UV distante.

As moléculas no estado excitado tem um tempo de vida finito, pois tal estado não é energeticamente o mais estável. Assim, um conjunto de moléculas que se encontre eletronicamente excitado tende a retornar ao estado eletrônico fundamental com a subseqüente liberação da energia que foi anteriormente adquirida com a absorção do fóton. A liberação de energia pode se dar na forma de calor ou como emissão de radiação eletromagnética. No caso de emissão de radiação, esta será chamada fluorescência quando houver conservação do spin eletrônico, e fosforescência quando o spin do elétron for alterado durante o tempo que a molécula permanece no estado excitado.

O retorno ao estado fundamental de um conjunto de moléculas excitadas segue, naturalmente, uma lei cinética de velocidade e a intensidade de emissão $\mathrm{I}_{\mathrm{E}}(\mathrm{t})$, é normalmente descrita como uma soma de funções exponenciais ${ }^{1}$ :

$$
I_{E}(t)=C+\sum_{i} A_{i} \exp \left[-\tau_{i} t\right]
$$

onde $A_{i}$ e $\tau_{i}$ são o fator pré-exponencial e o tempo de vida da componente i no estado excitado, respectivamente, e $C$ é uma constante. Assim, a evolução de um espectro de emissão ao longo do tempo tem um comportamento típico como o exibido na figura 1.

Tanto a intensidade de emissão quanto o tempo de vida de uma molécula eletronicamente excitada são afetados por uma série de fatores, tais como: taxas de decaimento radiativo, taxas de decaimento não radiativo, desativações colisionais, interações com o meio ou supressores, etc $^{1} \ldots$ Considerando-se os diversos

E-mail: tatvars@iqm.unicamp.br

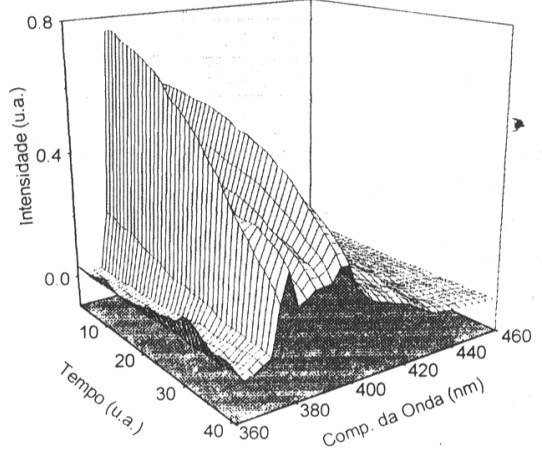

Figura 1. Decaimento, ao longo do tempo, da intensidade de emissão de um espectro hipotético.

tipos de processos unimoleculares, radiativos ou não, pode-se descrever o rendimento quântico de fosforescência como ${ }^{1,2}$ :

$\Phi_{F}=k_{s r} /\left(k_{s r}+k_{s n r}+k_{s t}+k_{s t}^{T}+k_{s n r}^{T}\right)$

sendo: $k_{s r}$ é a constante de emissão de fluorescência, $k_{s n r}$ é a constante de conversão interna independente da temperatuta, $k_{s t}$ é a constante de cruzamento intersistema independente da temperatura, $k_{s t}^{T}$ é a constante de cruzamento intersistema dependente da temperatura e $k_{s n r}^{T}$ é a constante de conversão interna dependente da temperatura. A intensidade de fluorescência, $I_{F}(t)$, em uma dada temperatura, pode ainda ser definida pela equação (3):

$I_{F}(t)=I_{o} \cdot k_{I} \cdot \Phi_{F}$

onde $I_{F}(t)$ é a intensidade de fluorescência na temperatura $t, I_{0}$ é a intensidade da luz de excitação e $k_{l}$ é uma constante de proporcionalidade que depende de condições instrumentais e da temperatura.

De modo análogo se pode definir equações para o rendimento quântico e intensidade de fosforescência, segundo as equações (4) e (5):

$\Phi_{T}=k_{t r} /\left(k_{t r}+k_{s n r}+k_{s t}+k_{s t}^{T}+k_{s n r}^{T}\right)$ 


$$
I_{T}(t)=I_{o} \cdot k_{2} \cdot \Phi_{T}
$$

Deve-se notar que várias das constantes de velocidade dependem da temperatura, de modo que este é um parâmetro importante na discussão das eficiências quânticas dos processos radiativos de emissão de fluorescência ou fosforescência.

Os métodos para a detecção e medida dos tempos de vida de estados excitados são divididos basicamente em três classes $^{3}$ : 1."Time Correlated Single Photon Counting" (TCSPC); 2. Integradores "Box Car" e métodos estroboscópicos; e 3. Métodos de resolução de fase.

O método TCSPC é estatístico e se baseia na probabilidade de que a emissão de um fóton por uma molécula excitada diminui exponencialmente com o decorrer do tempo; mede-se assim o tempo decorrido entre a excitação da amostra e a detecção do primeiro fóton que atinge o detector; este procedimento é repetido muitas vezes, de forma a se obter um histograma número de fótons $X$ tempo, o qual reproduz o comportamento temporal da emissão da amostra. Os métodos baseados no integrador "box car" e estroboscópios obtém a curva de decaimento de emissão através de amostragens da intensidade de emissão em diferentes períodos de tempo após a excitação da amostra. Nestes dois casos a excitação da amostra é realizada por meio de um pulso de luz, de forma que a resolução temporal do instrumento depende, entre outras coisas, da largura do pulso de excitação, ou seja, quanto menor a duração do pulso maior será a capacidade do instrumento resolver eventos de curta duração ${ }^{4}$.

Os métodos de resolução de fase se baseiam em um princípio menos óbvio do que os métodos anteriores: quando um conjunto de moléculas é excitado eletronicamente por um feixe de radiação com amplitude senoidalmente modulada à uma freqüência $\omega$, a intensidade de emissão dessa amostra também será modulada à mesma frequiência; porém, devido ao tempo de vida finito do estado excitado, a emissão da amostra estará defasada em relação à excitação. Portanto, esses métodos não são capazes de registrar a curva de decaimento de emissão em função do tempo, porém permitem o cálculo do tempo de vida radiativo de uma espécie com rapidez e eficiência ${ }^{3,5-8}$.

Para isto se considera que a intensidade de emissão experimental $I(t)$ é dada pela integral ${ }^{6}$ :

$$
\begin{aligned}
I_{t} & =\int_{0}^{t} \exp ^{\left(-\left[t-t^{\prime}\right] / \tau\right)}\left(I_{0}+I_{m} \cos (\omega k)\right) d t^{\prime} \\
& \text { e desde que } \mathrm{t} \gg>\tau
\end{aligned}
$$

$I_{0} \alpha t\left[I_{0}+I_{m} \cos d \cos (\omega t-d)\right]$

onde: $I_{0}$ é a intensidade da luz de excitação, $I_{m}$ é a amplitude da luz de excitação, $\omega$ é a frequência angular na qual o feixe de excitação está modulado e $\delta$ é a diferença de fase entre os dois feixes.

O tempo de vida radiativo da molécula no estado excitado pode ser determinado de duas maneiras: 1. por modulação relativa e 2. pelas medidas de diferença de fase. Tomando-se como base o esquema mostrado na figura 2 , a modulação relativa $(D)$ pode ser definida pela equação $(8)$ :

$$
D=\frac{\text { modulação da emissão }}{\text { modulação da excitação }}=\frac{\frac{\mathrm{H}_{4}-\mathrm{H}_{2}}{\mathrm{H}_{4}+\mathrm{H}_{2}}}{\frac{\mathrm{H}_{3}-\mathrm{H}_{1}}{\mathrm{H}_{3}+\mathrm{H}_{1}}}=\cos \delta=\left(1+\omega^{2} \tau^{2}\right)^{1 / 2}
$$

sendo que $\mathrm{H}_{1}, \mathrm{H}_{2}, \mathrm{H}_{3}$ e $\mathrm{H}_{4}$ são amplitudes absolutas dos sinais de excitação e de emissão, e estão representadas graficamente na figura 2 .

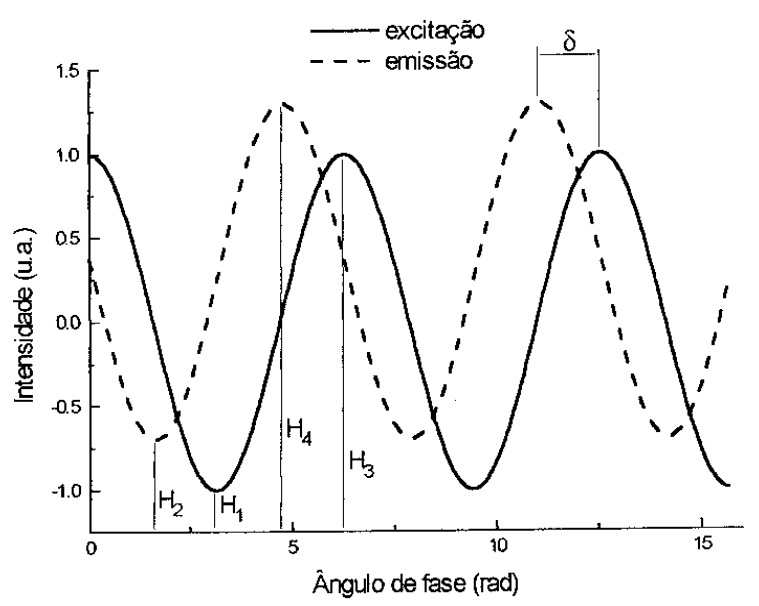

Figura 2. Representação da variação senoidal de um feixe de excitação e da emissão decorrente; estão representados também os parâmetros $\mathrm{H}_{1}, \mathrm{H}_{2}, \mathrm{H}_{3}$ e $\mathrm{H}_{4}$, explicados no texto.

Por outro lado, a diferença de fase $(\delta)$ entre a excitação e a emissão em uma dada freqüência angular $\omega$ é dada por:

$\tan \delta=\omega \tau$

onde $\tau$ é o tempo de vida da amostra no estado excitado.

Das equações (8) e (9) se pode verificar que a freqüência de modulação $\omega$ da luz de excitação é um fator determinante na sensibilidade e na resolução temporal de um instrumento com resolução de fase; o aumento ou a diminuição excessiva desta freqüência ocasionam uma diminuição tanto na modulação relativa $(D)$, como também na diferença de fase $(\delta)$, conforme ilustrado na figura $3^{3}$. Além disso, a equação (9) demonstra que para uma resolução de fase ótima a freqüência de modulação do sinal de excitação deve ser da mesma ordem de grandeza que o inverso do tempo de vida da amostra em estudo. Portanto, a freqüência ideal de modulação deve ser encontrada através de uma série preliminar de experimentos visando estimar o tempo de vida de fosforescência da amostra.

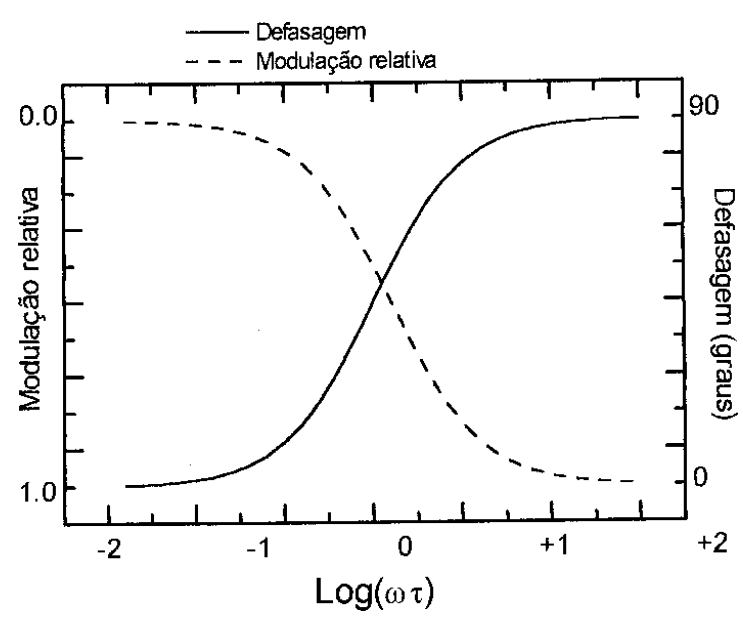

Figura 3. Variação da modulação relativa (D) $e$ da diferença de fase $(\delta)$ de um sinal de emissão em função do logarítmo da freqüência de modulação.

Para a geração de sinais com amplitude e/ou freqüência moduladas existem diversos dispositivos disponíveis, tais como: celas de Pockel, celas de Kerr; moduladores óptico-acústicos; 
descargas gasosas com modulação de rádio freqüência. e moduladores mecânicos (choppers) ${ }^{3}$. Com estes instrumentos é possível se obter sinais na faixa de freqüências de $\mathrm{mHz}$ à $\mathrm{GHz}$, e com isto se obter decaimentos de estados eletrônicos excitados em uma ampla escala temporal, de segundos à nanosegundos.

Para a detecção do sinal de emissão modulado é necessário o uso de detectores síncronos sensíveis à fase, como os amplificadores lock-in com dois canais ${ }^{7}$.

Neste trabalho é descrito um sistema simples para medidas de tempos de vida da ordem de milissegundos, usando-se uma fonte contínua de emissão, um obturador eletromecânico para excitação modulada da amostra e um amplificador lock-in como detector de mudança de fase. As medidas de tempo de vida da benzofenona (uma molécula fosforescente) dissolvida em poliestireno em diversas temperaturas foram usadas para estudar a dependência do tempo de vida com a temperatura do sistema e através desta dependência, os processos de relaxações do polímero.

\section{EXPERIMENTAL}

\subsection{Materiais utilizados}

Poliestireno (PS) da Poliestireno do Sul com $\mathrm{M}_{\mathrm{w}}=140.000 \mathrm{~g} /$ mol foi utilizado sem prévia purificação. Benzofenona (BZ) (Carlo-Erba) foi recristalizada duas vezes à partir da solução etanólica saturada. Os cristais foram secos à vácuo e armazenados ao abrigo da luz. Dicloroetano (Merck), com grau espectroscópico de pureza, foi utilizado sem prévia purificação.

\subsection{Preparação de amostras}

Para as medidas de emissão de fosforescência foi utilizada uma amostra de PS, na forma de filme, contendo $1 \%$ em massa de BZ. Para se preparar as amostras se dissolveu o PS e a BZ em dicloroetano em proporções adequadas; a solução foi colocada em uma placa de Petri e permitiu-se que o solvente fosse lentamente evaporado. O filme assim obtido tem $\approx 70 \mu \mathrm{m}$ de espessura, é transparente e homogêneo, sendo armazenado ao abrigo da luz e em dessecador até sua utilização.

\subsection{Equipamentos utilizados}

O sistema de instrumentos utilizado é composto por um espectrofluorímetro com vários módulos (fonte de luz, monocromador/fotomultiplicadora, sistema de controle de varredura informatizado e eletrômetro), e já foi descrito anteriormente. O sinal de saída do eletrômetro está conectado a um amplificador lock-in de dois canais (Stanford Research Systems, modelo SR-530), que controla um obturador de frequiência variável cuja função é modular a radiação de excitação. A freqüência de modulação foi selecionada em $150 \mathrm{~Hz}^{8}$. A excitação da amostra foi realizada com uma lâmpada de Xe de $150 \mathrm{~W}$ com comprimento de onda selecionado através de um monocromador de $25 \mathrm{~cm}$ de caminho óptico em $300 \mathrm{~nm}$.

O software de controle da instrumentação e aquisição de dados descrito anteriormente ${ }^{8}$ foi modificado de forma a incluir uma rotina para a aquisição de sinais de emissão com resolução de fase; esta rotina torna o instrumento capaz de adquirir um espectro de emissão com diferentes fases, resultando em uma superfície Intensidade X Comprimento de onda $X$ Ângulo de fase; e de controlar a temperatura entre 15 e $405 \mathrm{~K}$, com monitoramento da emissão em um único comprimento de onda e em várias fases de aquisição. Todos os dados gerados pelo instrumento são armazenados na forma de matrizes (para a superfície) e de tabelas Intensidade X Ângulo (para as varreduras de temperatura) em caracteres ASCII.

Neste trabalho foram realizados três tipos de experimentos, obtendo-se dados com a amostra de PS/BZ nas seguintes opções:
1. Obtenção dos espectros de fosforescência da BZ em diversas temperaturas (15 a 320K) sendo este experimento realizado com o amplificador lock-in ajustado para insensibilidade à fase do sinal.

2. Obtenção do sinal de fosforescência em $448 \mathrm{~nm}$ com controle da temperatura, e 36 fases de aquisição, de forma que a cada temperatura se adquira a intensidade de emissão de 0 a 360 graus, com uma variação de 10 graus.

3. Aquisição de um espectro completo de fosforescência (400 a $550 \mathrm{~nm}$ ) da amostra $\mathrm{PS} / \mathrm{BZ}$ à $75 \mathrm{~K}$ com resolução de fase de 0 a 360 graus. Neste caso, a cada comprimento de onda adquirido o monocromador é parado e a fase do amplificador é variada de 0 a 360 graus, sendo realizada uma aquisição de intensidade de fosforescência a cada 10 graus.

Para se obter os espectros nas diversas temperaturas a amostra foi termostatizada em um criostato, sendo que uma descrição detalhada do arranjo experimental foi feita anteriormente ${ }^{8}$.

Todo o procedimento necessário para a varredura da grade do monocromador, controle da freqüência de modulação, ajuste da fase do amplificador lock-in, controle da temperatura, armazenamento e tratamento dos resultados é realizado por um programa escrito em QuickBasic 4.5 rodando em um microcomputador PC-XT.

\section{RESULTADOS E DISCUSSÃO}

\subsection{Efeito da temperatura sobre o espectro de fosforescência}

A obtenção de espectros de fluorescência e de fosforescência de moléculas sonda em matrizes poliméricas em diversas temperaturas é um procedimento rotineiro em nosso laboratório, de forma que a interpretação dos dados gerados por este processo já é bem conhecida ${ }^{11,12}$. Pode-se desta forma, obter os espectros de excitação e de emissão de fosforescência da benzofenona em qualquer temperatura (entre 15 e $400 \mathrm{~K}$ ) e em quaisquer intervalos entre duas medidas. O tratamento posterior dos dados, realizado automaticamente pelo programa de controle do instrumento, pode fornecer dados de saída com diversas alternativas, entre elas: área total do espectro de emissão X temperatura (Figura 4a), intensidade em comprimentos de onda específicos X temperatura (Figura $4 \mathrm{~b}$ ) e relações entre intensidades de emissão em diferentes comprimentos de onda X temperatura. A análise de tais curvas permite correlacionar as modificações dos processos fotofísicos de desativação de fosforescência nas diversas temperaturas com os processos cooperativos de relaxação da matriz polimérica, no caso o $\mathrm{PS}^{9,10}$.
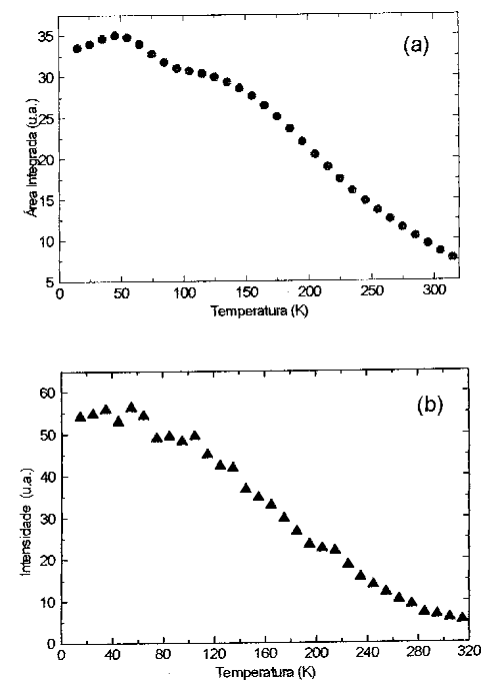

Figura 4. Área integrada de emissão versus temperatura (4.a) e intensidade de emissão em $448 n$ versus temperatura (4.b) para a BZ dissolvida em PS. 
A figura 4 mostra as variações da área integrada de fosforescência e da intensidade de fosforescência em $448 \mathrm{~nm}$ em função da temperatura da amostra de PS/BZ. Conforme se pode notar, existe uma pequena modificação no comportamento fotofísico por volta de $100 \mathrm{~K}$, porém o resultado é parcialmente obscurecido pelo ruído no sinal. Esta modificação no processo de desativação da benzofenona em poliestireno já foi reportada por outros autores ${ }^{11,12}$ e é atribuída aos movimentos dos anéis fenílicos do polímero que passam a ter energia térmica suficiente para a rotação em torno da ligação covalente com a cadeia polimérica. Esta rotação aumenta a probabilidade de desativações não radiativas da BZ excitada, através de transferência da energia de excitação da BZ para os anéis fenílicos da cadeia de PS.

\subsection{Determinação do tempo de vida de fosforescência}

A fim de se verificar se a resolução de fases poderia gerar bons resultados espectrais com a presente amostra, foi realizado um experimento com a subrotina de aquisição de espectro de fosforescência com resolução de fase, o espectro resultante é mostrado na figura 5, para a temperatura de $75 \mathrm{~K}$. Nessa figura se observa que o sinal de intensidade de fosforescência apresenta valores negativos em alguns ângulos de fase, o que ocorre porque a magnitude dos sinais medidos pelo amplificador depende do cosseno do ângulo de fase, de maneira que os valores negativos não devem ser interpretados como uma "emissão negativa", mas sim como um artifício matemático gerado pela instrumentação. A partir da superfície mostrada na figura 5 foi escolhido o comprimento de onda fixo de emissão em 448nm para a realização da varredura de temperatura com aquisição de tempos de vida. Este comprimento de onda foi escolhido pois é nesta região que acontece a maior variação na intensidade do sinal com a variação da fase.

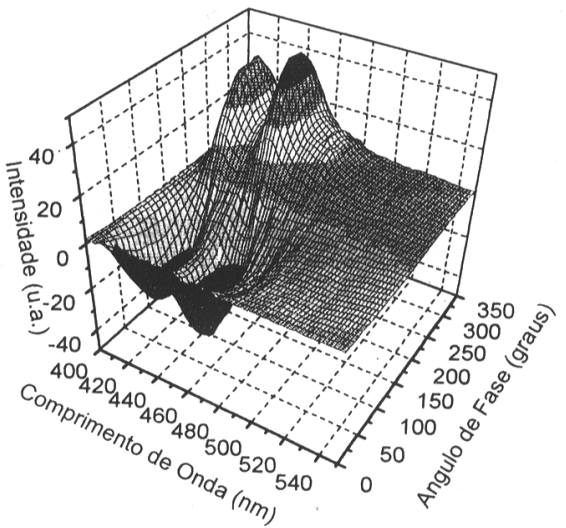

Figura 5. Superfície de fosforescência com resolução de fase da amostra de $P S / B Z$ a $75 K$.

O experimento de varredura de temperatura com resolução de fase consiste no seguinte procedimento: a amostra é refrigerada no interior do criostato até $15 \mathrm{~K}$; excita-se a mesma com o feixe modulado em $150 \mathrm{~Hz}$; focaliza-se o sinal de espalhamento de excitação na fenda do detector e mede-se a fase deste sinal de espalhamento; um comando é enviado ao amplificador de forma a tornar a fase medida como um "ângulo de referência"; a seguir o monocromador de detecção é sintonizado para $448 \mathrm{~nm}$, quando então realiza-se a medida da intensidade de emissão neste comprimento de onda em 36 ângulos diferentes de aquisição $(0$ a 360 graus, em intervalos de 10 graus); a temperatura é então aumentada para $25 \mathrm{~K}$ e o processo é repetido até que a temperatura final $(320 \mathrm{~K})$ seja alcançada.

A cada temperatura é gerada uma curva intensidade $X$ ângulo; essa curva tem a forma cossenoide, pois o ângulo de espalhamento é considerado como zero, de forma que a regressão cossenoidal da curva nos fornece o ângulo de defasagem da emissão em relação ao ângulo de excitação. A equação cossenoidal utilizada para se ajustar as curvas é dada por:

$I_{(x)}=I_{0} \cos (x+\alpha)+C$

onde $I_{0}$ e $C$ são parâmetros de ajuste, e $\alpha$ é o ângulo de defasagem.

$\mathrm{Na}$ figura 6 está representada a curva cossenoide da emissão em $448 \mathrm{~nm}$ e a $15 \mathrm{~K}$, e também a curva obtida com a regressão cossenoidal. Nesse caso, o ângulo de defasagem foi determinado como sendo de 311,9 graus e usando-se este valor e o valor da freqüência utilizada no modulador de $150 \mathrm{~Hz}$ na equação 9, obtém-se um tempo de vida $\tau=7,4 \mathrm{~ms}$. Destaca-se, ainda, que a freqüência do modulador não foi escolhida ao acaso; note-se que $1 / 150 \mathrm{~Hz} \approx 0,007 \mathrm{~s}$, ou seja, a freqüência de modulação é muito próxima do inverso do tempo de vida esperado para a amostra, de acordo com a figura 3 .

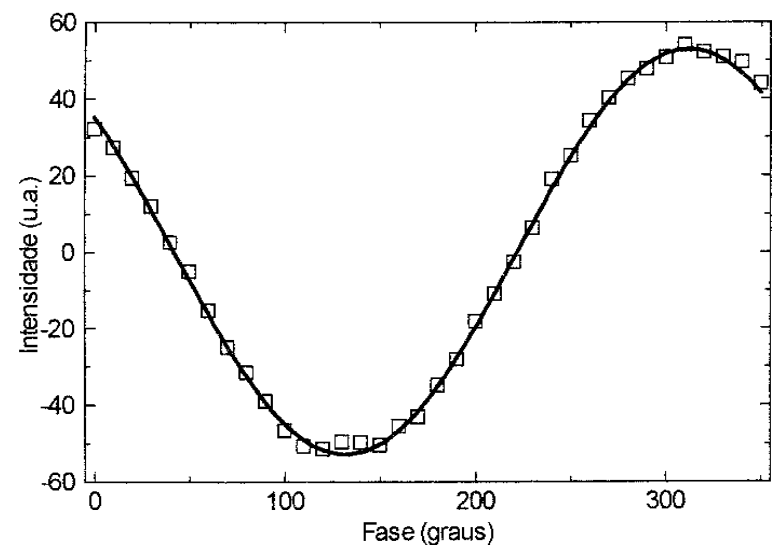

Figura 6. Intensidade de fosforescência em 448nm versus ângulo de fase do detector da amostra de PS/BZ, a $15 \mathrm{~K}$.

A repetição do cálculo do tempo de vida para todas as outras medidas em temperaturas diferentes permite a construção de uma curva tempo de vida X temperatura (Figura 7). Como se pode ver na curva de tempos de vida em função da temperatura tem-se que até $100 \mathrm{~K}$ o tempo de vida de fosforescência é aproximadamente constante $(7,4 \mathrm{~ms})$, e após esta temperatura existe uma diminuição monotônica do tempo de vida, evidenciando que a desativação das moléculas de BZ excitadas eletronicamente se torna mais eficiente.

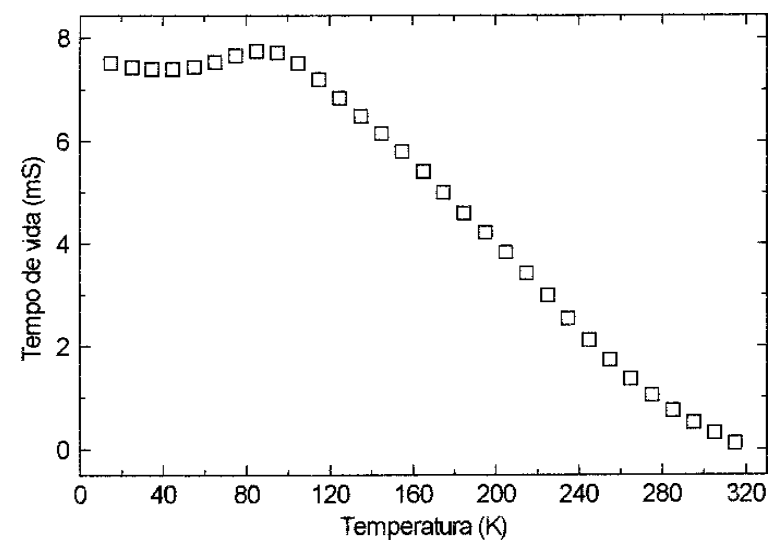

Figura 7. Tempos de vida de fosforescência da BZ em PS, em função da temperatura. 
O tempo de vida da benzofenona em matrizes sólidas já foi determinado por outros autores ${ }^{13-15}$ e se encontra na faixa de 5-10 milissegundos ${ }^{9}$, sendo, portanto, o valor determinado neste trabalho coerente com os reportados. Estes autores têm demonstrado também que o tempo de vida sofre modificações com a temperatura e é grandemente influenciado pelos processos de relaxações da matriz.

\section{CONCLUSÕES}

A espectroscopia de luminescência com resolução de fases mostrou-se um método simples para o estudo dos processos de emissão de luminescência de sondas dissolvidas em matrizes poliméricas, permitindo, no caso de sua dependência com a temperatura, que estudos de processos de relaxações de polímeros possam ser realizados. No sistema implantado em nosso laboratório o controle do experimento é feito com um amplificador lock-in, de modo que os limites de detecção de tempos de vida estão na faixa de mili-segundos. Um programa de controle de todos os instrumentos foi especialmente escrito, permitindo a realização de experimentos diferentes com o mesmo conjunto de aparelhos, tornando, pois, o arranjo experimental muito flexível. Os dados são gerados em caracteres ASCII de modo que programas usuais de tratamento de dados podem ser utilizados.

\section{AGRADECIMENTOS}

FAPESP, FINEP e PADCT/CNPq pelo apoio financeiro. M. $T$. agradece a FAPESP pela bolsa de doutorado.

\section{REFERÊNCIAS}

1. Birks, J. B.; Photophysics of Aromatic Molecules; WilleyInterscience, London, 1970.

2. Sakurovs, R. and Ghiggino, K. P.; Aust. J. Chem. 1981, 34, 1367.

3. Rabek, J. F.; Experimental Methods in Photochemistry and Photophysics; John Willey \& Sons, Avon, 1982.

4. O'Connor, D. and Phillips, D.; Time-Correlated Single Photon Counting; Academic Press, London, 1984.

5. Bailey, E. A. and Rollefson, G. K.; J. Chem. Phys. 1954, $21,1315$.

6. McGown, L. B. and Bright, F. V.; Anal. Chem. 1984, 56, 1400A.

7. Manual de operação do amplificador lock-in SR530, Stanford Research Systems, 1989.

8. Talhavini, M. e Atvars, T. D. Z.; Quím. Nova 1995, 18, 298.

9. Sabadini, E.; Tese de Mestrado; UNICAMP, 1988.

10. Talhavini, M.; Dissertação de Mestrado; UNICAMP, 1995.

11. Guillet, J. E., em: Advances in Photochemistry, Volman, D. H.; Hammond, G. S. and Gollnick, K. (editores:), Wiley, New York, Vol. 14. P. 911988.

12. Halary, J. L. and Monnerie, L., em: Photophysical and Photochemical Tools in Polymer Science, NATO ASI Series, Winnik, M. (editor), D. Riedel, New York, Vol. 182, p. 589, 1986.

13. Rusakonics, R.; Byer, G. W. e Leermakers, P. A.; J. Am. Chem. Soc. 1971, 93, 3263.

14. Hikosaka, Y. and Hikida, T.; Chem. Phys. 1996, 203, 137.

15. Zevenhuijzen, D. and Van Der Werf, R.; Chem. Phys. 1977, 26, 279. 